\title{
NEW-HOPLA
}

\section{a higher-order process language with name generation}

\author{
Glynn Winskel \\ Computer Laboratory. University of Cambridge, UK \\ Francesco Zappa Nardelli \\ INRIA \& Computer Laboratory, University of Cambridge, UK
}

\begin{abstract}
This paper introduces new-HOPLA, a concise but powerful language for higherorder nondeterministic processes with name generation. Its origins as a metalanguage for domain theory are sketched but for the most part the paper concentrates on its operational semantics. The language is typed, the type of a process describing the shape of the computation paths it can perform. Its transition semantics, bisimulation, congruence properties and expressive power are explored. Encodings are given of well-known process algebras, including $\boldsymbol{\pi}$-calculus, Higher-Order $\boldsymbol{\pi}$-calculus and Mobile Ambients.
\end{abstract}

\section{The origins of new-HOPLA}

This work is part of a general programme (reported in [8]), to develop a domain theory which scales up to the intricate languages, models and reasoning techniques used in distributed computation. This ambition led to a concentration on path based models, and initially on presheaf models because they can even encompass causal dependency models like event structures; so 'domains' is being understood more broadly than usual, to include presheaf categories.

The general methodology has been to develop domain theories with a rich enough life of their own to suggest powerful metalanguages. The point to emphasise is that in this way informative domain theories can have a pro-active role; they can yield new metalanguages, by their nature very expressive, accompanied by novel ways to deconstruct existing notions into more primitive ones, as well as new analysis techniques. A feature of presheaf models has been very useful: in key cases there is often a strong correspondence between elements of the presheaf denotation and derivations in an operational semantics. In the cases of HOPLA and new-HOPLA the presheaf models have led not only the core operations of the language, and a suitable syntax, but also to their operational semantics.

This paper reports on new-HOPLA, a compact but expressive language for higherorder nondeterministic processes with name generation. It extends the language HOPLA of Nygaard and Winskel [7] with name generation, and like its predecessor has its origins in a domain theory for concurrency. Specifically it arose out of the metalan- 
guage implicitly being used in giving a presheaf semantics to the $\pi$-calculus [2]. But a sketch of its mathematical origins and denotational semantics does not require that heavy an investment, and can be based on path sets rather than presheaves. ${ }^{1}$

The key features of new-HOPLA hinge on its types and these can be understood independently of their origin as objects, and constructions on objects, in a category of domains - to be sketched shortly within the simple domain theory of path sets. A type $\mathbb{P}$ specifies the computations possible with respect to a given current set of names; if a process has type $\mathbb{P}$, then any computation path it performs with the current set of names $\boldsymbol{s}$ will be an element of $\mathbb{P}(\boldsymbol{s})$.

A central type constructor is that of prefix type $\mathbb{P}$; at a current set of names $\boldsymbol{s}$, a process of this type $! \mathbb{P}$, if it is to do anything, is constrained to first doing a prototypical action ! before resuming as a process of type $\mathbb{P}$. (Actions within sum or tensor types will come to be tagged by injections and so of a less anonymous character.)

In the category of domains, domains can be tensored together, a special case of which gives us types of the form $\mathbb{N} \otimes \mathbb{P}$, a kind of dynamic sum which at current names $\boldsymbol{s}$ comprises paths of $\mathbb{P}(\boldsymbol{s})$ tagged by a current name which serves as an injection function. There is also a more standard sum $\boldsymbol{\Sigma}_{\boldsymbol{i} \in I} \mathbb{P}_{\boldsymbol{i}}$ of an indexed family of types $\mathbb{P}_{\boldsymbol{i}}$ where $i \in I$; this time paths are tagged by indices from the fixed set $I$ rather than the dynamic set of names.

The remaining type constructions are the formation of recursive types, and three forms of function space. One is a 'linear function space' $\mathbb{N} \rightarrow \mathbb{P}$, the type of processes which given a name return a process of type $\mathbb{P}$. Another is a 'continuous function space' $\mathbb{P} \rightarrow \mathbb{Q}$, the type of processes which given a process of type $\mathbb{P}$ return a process of type $\mathbb{Q}$. There is also a type $\delta \mathbb{P}$ associated directly with new-name generation. A process of type $\delta \mathbb{P}$ takes any new name (i.e. a name not in the current set of names) as input and returns a process of type $\mathbb{P}$. Name generation is represented by new name abstraction, to be thought of as picking a new name (any new name will do as well as any other), and resuming as a process in which that new name is current.

This summarises the rather economical core of new-HOPLA. Very little in the way of standard process algebra operations are built in - nothing beyond a prefix operation and nondeterministic sum. By being based on more fundamental primitives than usual, the language of new-HOPLA is remarkably expressive. As additional motivation we now turn to how these primitives arise from a mathematical model refining the intuitions we have just presented.

A domain theory If for the moment we ignore name generation, a suitable category of domains is that of Lin. Its objects, path orders, are preorders $\mathbb{P}$ consisting of computation paths with the order $\boldsymbol{p} \leq \boldsymbol{p}^{\boldsymbol{\prime}}$ expressing how a path $\boldsymbol{p}$ extends to a path $\boldsymbol{p}^{\prime}$. A path order $\mathbb{P}$ determines a domain $\widehat{\mathbb{P}}$, that of its path sets, left-closed sets w.r.t. $\leq_{\mathbf{P}}$, ordered by inclusion. (Such a domain is a prime-algebraic complete lattice, in which the complete primes are precisely those path sets generated by individual paths.) The arrows of Lin, linear maps, from $\mathbb{P}$ to $\mathbb{Q}$ are join-preserving functions from $\widehat{\mathbb{P}}$ to $\widehat{\mathbb{Q}}$.

\footnotetext{
${ }^{1}$ Path sets arise by 'flattening' presheaves, which can be viewed as characteristic functions to truth values given in the category of sets, as sets of realisers, to simpler characteristic functions based on truth values $0 \leq 1[8]$.
} 
The category Lin is monoidal-closed with a tensor given by the product $\mathbb{P} \times \mathbf{Q}$ of path orders and a corresponding function space by $\mathbb{P}^{\text {oD }} \times \mathbb{Q}$-it is easy to see that joinpreserving functions from $\widehat{\mathbb{P}}$ to $\widehat{\mathbb{Q}}$ correspond to path sets of $\mathbb{P}^{\text {op }} \times \mathbf{Q}$. In fact Lin has enough structure to form a model of Girard's classical linear logic [4]. To exhibit its exponential! we first define the category Cts to consist, like Lin, of path orders as objects but now with arrows the Scott-continuous functions between the domains of path sets. The inclusion functor Lin $\hookrightarrow$ Cts has a left adjoint ! : Cts $\rightarrow$ Lin which takes a path order $\mathbb{P}$ to a path order consisting of finite subsets of $\mathbb{P}$ with order

\section{$P \leq \mid \mathrm{P} P^{\prime}$ iff $\forall p \in P \exists p^{\prime} \in P^{\prime} . p \leq_{\mathrm{P}} p^{\prime}$}

- so $! \mathbb{P}$ can be thought of as consisting of compound paths associated with several runs.

The higher-order process language HOPLA is built around constructions in the category Lin. Types of HOPLA, which may be recursively defined, denote objects of Lin, path orders circumscribing the computation paths possible. As such all types support operations of nondeterministic sum and recursive definitions, both given by unions. Sum types are provided by coproducts, and products, of Lin, both given by the disjoint juxtaposition of path orders; they provide injection and projection operations. There is a type of functions from $\mathbb{P}$ to $\mathbb{Q}$ given by $(\mathbb{I})^{\mathbf{o p}} \times \mathbf{Q}$, the function space of Cts; this gives the operation of application and lambda abstraction. To this the adjunction yields a primitive prefix operation, a continuous map $\mathbb{P} \rightarrow ! \mathbb{P}$, given by the unit at $\mathbb{P}$; it is accompanied by a destructor, a prefix-match operation, obtained from the adjunction's natural isomorphism. For further details, encodings of traditional process calculi in HOPLA and a full abstraction result, the reader is referred to [7, 9].

A domain theory for name generation We are interested in extending HOPLA to allow name generation. We get our inspiration from the domain theory. As usual a domain theory for name generation is obtained by moving to a category in which standard domains are indexed functorially by the current set of names. The category $\mathcal{I}$ consists of finite sets of names related by injective functions. The functor category $\operatorname{Lin}^{\mathcal{I}}$ has as objects functors $\mathbb{P}: \mathcal{I} \rightarrow \mathbf{L i n}$, so path orders $\mathbb{P}(\boldsymbol{s})$, indexed by finite sets of names $\boldsymbol{s}$, standing for the computation paths possible with that current set of names; its arrows are natural transformations $\alpha=\left\langle\alpha_{s}\right\rangle_{s \in \mathcal{I}}: \mathbb{P} \rightarrow \mathbb{Q}$, with components in Lin One important object in $\mathbf{L i n}^{\mathcal{T}}$ is the object of names $\mathbf{N}$ providing the current set of names, so $\mathbf{N}(\boldsymbol{s})=\boldsymbol{s}$ regarded as a discrete order, at name set $\boldsymbol{s}$. Types of new-HOPLA will denote objects of $\mathbf{L i n}^{\mathcal{I}}$.

The category has coproducts and products, both given by disjoint juxtaposition at each component. These provide a sum type $\boldsymbol{\Sigma}_{\boldsymbol{i} \in \boldsymbol{I}} \mathbb{P}_{\boldsymbol{i}}$ from a family of types $\left(\mathbb{P}_{\boldsymbol{i}}\right)_{\boldsymbol{i} \in \boldsymbol{I}}$. It has injections producing a term $i$ :t of type $\boldsymbol{\Sigma}_{\boldsymbol{i} \in \boldsymbol{I}} \boldsymbol{P}_{\boldsymbol{i}}$ from a term $\boldsymbol{t}$ of type $\mathbb{P}_{\boldsymbol{i}}$, for $\boldsymbol{i} \in \boldsymbol{I}$. Projections produce a term $\boldsymbol{\pi}_{\boldsymbol{i}} \boldsymbol{t}$ of type $\mathbb{P}_{\boldsymbol{i}}$ from a term $\boldsymbol{t}$ of the sum type.

There is a tensor not pointwise from the tensor of Lin. Given $\mathbb{P}$ and $\mathbb{Q}$ in $\mathbf{L i n}^{\mathcal{I}}$ we define $\mathbb{P} \otimes \mathbb{Q}$ in $\operatorname{Lin}^{\mathcal{I}}$ so that $(\mathbb{P} \otimes \mathbb{Q})(\boldsymbol{s})=\mathbb{P}(\boldsymbol{s}) \times \mathbb{Q}(\boldsymbol{s})$ at $\boldsymbol{s} \in \mathcal{I}$. We will only use a special case of this construction to form tensor types $\mathbb{N} \otimes \mathbb{P}$, so $(\mathbb{N} \otimes \mathbb{P})(s)=s \times \mathbb{P}(s)$ at $s \in \mathcal{I}$. These are a form of 'dynamic sum', referred to earlier, in which the components and the corresponding injections grow with the availability of new names. There are term constructors producing a term $\boldsymbol{n} \cdot \boldsymbol{t}$ of type $\mathbf{N} \otimes \mathbb{P}$ from a term $\boldsymbol{t}$ of type $\mathbb{P}$ and a 
name $n$. There are projections $\pi_{n} t$ forming a term of type $\mathbb{P}$ from a term $t$ of tensor type.

At any stage $s$, the current set of names, a new name can be generated and used in a term in place of a variable over names. This leads to the central idea of new-name abstractions of type $\delta \mathbb{P}$ where $\delta \mathbb{P}(s)=\mathbb{P}(\boldsymbol{s} \cup\{\star\})$ at name set $\boldsymbol{s}$. As observed by Stark [14] the construction $\delta \mathbb{P}$ can be viewed as a space of functions from $\mathbb{N}$ to $\mathbb{P}$ but with the proviso that the input name is fresh. A new-name abstraction is written new $\alpha . t$ and has type $\delta \mathbb{P}$, where $t$ is a term of type $\mathbb{P}$. New-name application is written $t[n]$, where $t$ has type $\delta \mathbb{P}$, and requires that the name $n$ is fresh w.r.t. the names of $t$.

The adjunction $\operatorname{Lin} \stackrel{\stackrel{1}{\perp}}{\leftrightarrows}$ Cts induces anadjunction $\mathbf{L i n}^{\mathcal{I}} \stackrel{\frac{1}{\perp}}{\leftrightarrows}$ Cts $^{\mathcal{I}}$ where the left adjoint is got by extending the original functor !: Cts $\rightarrow$ Lin in a pointwise fashion. The unit of the adjunction provides a family of maps from $\mathbb{P}$ to $! \mathbb{P}$ in $\mathbf{C t s}^{\mathcal{I}}$. As with HOPLA, these yield a prefix operation $! t$ of type $! \mathbb{P}$ for a term $t$ of type $\mathbb{P}$. A type of the form $! \mathbb{P}$ is called a prefix type; its computation paths at any current name set first involve performing a prototypical action, also called '!'.

To support higher-order processes we need function spaces $\mathbb{P} \rightarrow \mathbb{Q}$ such that

$$
\operatorname{Lin}^{\mathcal{I}}(\mathbb{R}, \mathbb{P} \longrightarrow \mathbb{Q}) \cong \operatorname{Lin}^{\mathcal{I}}(\mathbb{R} \otimes \mathbb{P}, \mathbb{Q})
$$

natural in $\mathbb{R}$ and $\mathbb{Q}$. Such function spaces do not exist in general-the difficulty is in getting a path order $\mathbb{P} \multimap \mathbb{Q}(\boldsymbol{s})$ at each name set $\boldsymbol{s}$. However a function space $\mathbb{P} \multimap \mathbb{Q}$ does exist in the case where $\mathbb{P} f$ preserves complete primes and $\mathbb{Q} f$ preserves nonempty meets for each map $f: s \rightarrow s^{\prime}$ in $\mathcal{I}$. This suggests limiting the syntax of types to special function spaces $\mathbb{N} \rightarrow \mathbb{Q}$ and $! \mathbb{P} \multimap \mathbb{Q}$, the function space in $\mathbf{C t s}^{\mathcal{I}}$. The function spaces are associated with operations of application and lambda abstraction.

Related work and contribution The above domain theoretic constructions provide the basis of new-HOPLA. It resembles, and indeed has been inspired by, the metalanguages for domain theories with name generation used implicitly in earlier work $[3,14,2]$, as well as the language of FreshML [11]. The language new-HOPLA is distinguished through the path-based domain theories to which it is fitted and, as we will see, in itself forming a process language with an operational semantics. For lack of space, in this extended abstract we omit the proofs of the theorems; these can be found in [16].

\section{The language}

Types The type of names is denoted by $\mathbb{N}$. The types of processes are defined by the grammar below.

$\mathbb{P}::=\mathbf{0}|\mathbb{N} \otimes \mathbb{P}| ! \mathbb{P}|\delta \mathbb{P}| \mathbb{N} \rightarrow \mathbb{P}|\mathbb{P} \rightarrow \mathbb{Q}| \Sigma_{i \in I} \mathbb{P}_{i}\left|\mu_{j} P_{1} \ldots P_{k} .\left(\mathbb{P}_{1} \ldots \mathbb{P}_{k}\right)\right| P$

The sum type $\Sigma_{i \in I} \mathbb{P}_{i}$ when $I$ is a finite set, is most often written $i_{1}: \mathbb{P}+\cdots+i_{k}: \mathbb{P}$. The symbol $P$ is drawn from a set of type variables used in defining recursive types; closed type expressions are interpreted as path orders. The type $\mu_{j} P_{1} \ldots P_{k}\left(\mathbb{P}_{1} \ldots \mathbb{P}_{k}\right)$ is interpreted as the $j$-component, for $1 \leq j \leq k$, of the 'least' solution to the defining equations $P_{1}=\mathbb{P}_{1}, \ldots, P_{k}=\mathbb{P}_{k}$, where the expressions $\mathbb{P}_{1} \ldots \mathbb{P}_{k}$ may contain the $P_{j}$ 's. 


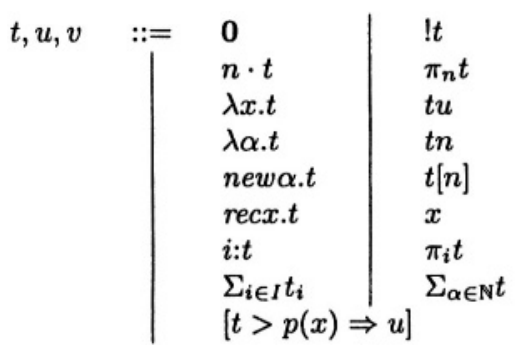

inactive process and prototypical action tensor and projection process abstraction and application name abstraction and application new-name abstraction and application recursive definition and process variables injection and projection sum and sum over names pattern matching

\section{Table 1. new-HOPLA: syntax of terms}

Terms and actions We assume a countably infinite set of name constants, ranged over by $a, b, \ldots$ and a countably infinite set of name variables, ranged over by $\alpha, \beta, \ldots$ Names, either constants or variables, are ranged over by $m, n, \ldots$. We assume an infinite, countable, set of process variables, ranged over by $x, y, \ldots$

Every type is associated with actions processes of that type may do. The actions are defined by the grammar below:

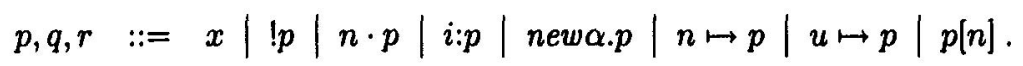

As we will see shortly, well-typed actions are constructed so that they involve exactly one prototypical action! and exactly one 'resumption variable' $\boldsymbol{x}$. Whenever a term performs the action, the variable of the action matches the resumption of the term: the typings of an action thus relates the type of a term with the type of its resumption. According to the transition rules a process of prefix type $\mathbb{P}$ may do actions of the form $! p$, while a process of tensor or sum type may do actions of the form $\boldsymbol{n} \cdot \boldsymbol{p}$ or $\boldsymbol{i}: \boldsymbol{p}$ respectively. A process of type $\delta \mathbb{P}$ does actions of the form new $\alpha . p$ meaning that at the generation of a new name, $\boldsymbol{a}$ say, as input the action $\boldsymbol{p}[\boldsymbol{a} / \boldsymbol{\alpha}]$ is performed. Actions of function type $\boldsymbol{n} \mapsto \boldsymbol{p}$ or $\boldsymbol{u} \mapsto \boldsymbol{p}$ express the dependency of the action on the input of a name $\boldsymbol{n}$ or process $\boldsymbol{u}$ respectively. The final clause is necessary in building up actions because we sometimes need to apply a resumption variable to a new name.

The terms are defined by the grammar reported in Table 1.

In new-HOPLA actions are used as patterns in terms $[t>p(x) \Rightarrow u]$ where we explicitly note the resumption variable $\boldsymbol{x}$. If the term $\boldsymbol{t}$ can perform the action $\boldsymbol{p}$ the resumption of $\boldsymbol{t}$ is passed on to $\boldsymbol{u}$ via the variable $\boldsymbol{x}$.

We assume an understanding of the free name variables (the binders of name variables are $\lambda \boldsymbol{\alpha}_{-}-$, new $\boldsymbol{\alpha}_{.}-$, and $\boldsymbol{\Sigma}_{\boldsymbol{\alpha} \in \mathbb{N}^{-}}$) and of the free process variables (the binders of process variables are $\lambda x$. , and $[t>p(x) \Rightarrow-]$ ) of a term, and of substitutions. The support of a closed term, denoted $n(t)$, is the set of its name constants. We say that a name $n$ is fresh for a closed term $t$ if $n \notin n(t)$.

Transition rules The behaviour of terms is defined by a transition relation of the form

$$
s \vdash t \stackrel{p(x)}{\longrightarrow} t^{\prime}
$$

where $\boldsymbol{s}$ is a finite set of name constants such that $\mathbf{n}(t) \subseteq \boldsymbol{s}$. The transition above should be read as 'with current names $\boldsymbol{s}$ the term $\boldsymbol{t}$ can perform the action $\boldsymbol{p}$ and resume as $t^{\prime \prime}$. We generally note the action's resumption variable in the transitions; 


$$
\begin{aligned}
& \frac{\mathbb{P} ; s \vdash t_{i} \stackrel{p(x)}{\longrightarrow} t^{\prime}}{! \mathbb{P} ; s \vdash ! t \stackrel{\mid x(x)}{\longrightarrow} t} \quad \frac{\mathbb{P} ; s \vdash t[a / \alpha] \stackrel{p(x)}{\longrightarrow} u \quad a \in s}{\mathbb{P} ; s \vdash \Sigma_{i \in I} t_{i} \stackrel{p(x)}{\longrightarrow} t^{\prime}} \quad \frac{p(x)}{\mathbb{P} ; s \vdash \Sigma_{\alpha \in \mathbb{N}} t \stackrel{p}{\longrightarrow} u} \\
& \frac{\mathbb{P} ; s \vdash t \stackrel{p(x)}{\longrightarrow} t^{\prime} \quad a \in s}{\mathbb{N} \otimes \mathbb{P} ; s \vdash a \cdot t \stackrel{a \cdot p(x)}{\longrightarrow} t^{\prime}} \quad \frac{\mathbb{N} \otimes \mathbb{P} ; s \vdash t \stackrel{a \cdot p(x)}{\longrightarrow} t^{\prime}}{\mathbb{P} ; s \vdash \pi_{a} t \stackrel{p(x)}{\longrightarrow} t^{\prime}} \quad \frac{\mathbb{P} ; s \vdash t[\text { recy.t } / y] \stackrel{p(x)}{\longrightarrow} u}{\mathbb{P} ; s \vdash \text { recy.t } \stackrel{p(x)}{\longrightarrow} u} \\
& \frac{\mathbb{P}_{i} ; s \vdash t \stackrel{p(x)}{\longrightarrow} t^{\prime}}{\Sigma_{i \in I} \mathbb{P}_{i} ; s \vdash i: t \stackrel{i: p(x)}{\longrightarrow} t^{\prime}} \quad \frac{\Sigma_{i \in I} \mathbb{P}_{i} ; s \vdash t \stackrel{i: p(x)}{\longrightarrow} t^{\prime}}{\mathbb{P}_{i} ; s \vdash \pi_{i} t \stackrel{p(x)}{\longrightarrow} t^{\prime}} \quad \stackrel{\mathbb{Q} ; s \vdash t[u / x] \stackrel{p(x)}{\longrightarrow} v \quad s \vdash u: \mathbb{P}}{\mathbb{P} \rightarrow \mathbb{Q} ; s \vdash \lambda x . t \stackrel{u \mapsto p(x)}{\longrightarrow} v}
\end{aligned}
$$

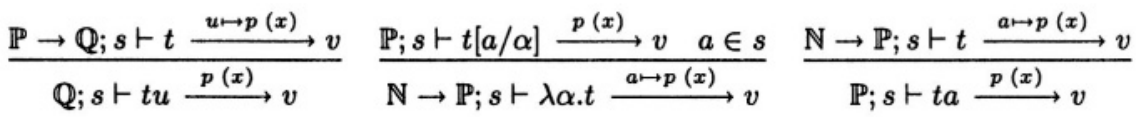

$$
\begin{aligned}
& \frac{\mathbb{P} ; s \dot{\cup}\{a\} \vdash t[a / \alpha] \stackrel{p[a / \alpha](x)}{\longrightarrow} u[a / \alpha]}{\delta \mathbb{P} ; s \vdash \text { new } \alpha . t \stackrel{\text { new } \alpha . p\left[x^{\prime}[\alpha] / x\right]\left(x^{\prime}\right)}{\longrightarrow} \text { new } \alpha . u} \quad \frac{\delta \mathbb{P} ; s \vdash t \stackrel{\text { new } \alpha . p\left[x^{\prime}[\alpha] / x\right]\left(x^{\prime}\right)}{\longrightarrow} u}{\mathbb{P} ; s \cup\{a\} \vdash t[a] \stackrel{p[a / \alpha](x)}{\longrightarrow} u[a]} \\
& \frac{\mathbb{P} ; s \vdash t \stackrel{p(x)}{\longrightarrow} t^{\prime} \quad \mathbb{Q} ; s \vdash u\left[t^{\prime} / x\right] \stackrel{q\left(x^{\prime}\right)}{\longrightarrow} v}{q\left(x^{\prime}\right)}
\end{aligned}
$$

In the rule for new name abstraction, the conditions $a \notin n(p)$ and $a \notin n(u)$ must hold.

Table 2. new-HOPLA: transition rules

this simplifies the transition rules in which the resumption variable must be explicitly manipulated.

So the transition relation is given at stages indexed by the set of current names $\boldsymbol{s}$. The body of an abstraction over names $\lambda \alpha . t$ can only be instantiated with a name in $\boldsymbol{s}$, and an abstraction over processes $\lambda \boldsymbol{x}$.t can only be instantiated with a process whose support is contained in $\boldsymbol{s}$. As the transition relation is indexed by the current set of names, it is possible to generate new names at run-time. Indeed, the transition rule for new-name abstraction new $\boldsymbol{\alpha}$.t extends the set $\boldsymbol{s}$ of current names with a new name $\boldsymbol{a} \notin \boldsymbol{s}$; this name $\boldsymbol{a}$ is then passed to $t$ via the variable $\boldsymbol{\alpha}$. The transition rules must respect the typings of actions and terms given in the next section. Formally:

Definition 1 (Transition relation) For closed terms $\boldsymbol{t}$ such that $\boldsymbol{s} \vdash \boldsymbol{t}: \mathbb{P}$ and path patterns such that $s ; ; x: \mathbb{Q} \Vdash p: \mathbb{P}$ the rules reported in Table 2 define a relation $\mathbb{P} ; \boldsymbol{s} \vdash t \stackrel{p(x)}{\longrightarrow} u$, called the transition relation.

Typing judgements Consider a term $t=t^{\prime}[\alpha]$. As we have discussed in the previous section, this denotes a new-name application: any name instantiating $\alpha$ should be fresh for the term $t^{\prime}$. Consider now the context $C[-]=\lambda \alpha_{.}-$. In the term $C[t]=\lambda \alpha \cdot\left(t^{\prime}[\alpha]\right)$, the variable $\alpha$ is abstracted via a lambda abstraction, and may be instantiated with any current name. In particular it may be instantiated with names that belong to the support of $\boldsymbol{t}^{\prime}$, thus breaking the hypothesis that $t^{\prime}$ has been applied to a fresh name. The same problem arises with contexts of the form $C[-]=\Sigma_{\alpha \in \mathbf{N}}-$. 
Moreover, if the process variable $x$ is free in $t$, a context like $C[-]=\lambda x .-$ might replace $x$ with an arbitrary term $\boldsymbol{u}$. As the name instantiating $\boldsymbol{\alpha}$ might belong to the support of $u$, nothing ensures it is still fresh for the term $t[u / x]$.

The type system must sometimes ensure that name variables are instantiated by fresh names. To impose this restriction, the typing context contains not only typing assumptions about name and process variables, such as $\alpha: \mathbb{N}$ and $x: \mathbb{P}$, but also freshness assumptions about them, written $(\alpha, \beta)$ or $(\alpha, x)$. The intended meaning of $(\alpha, \beta)$ is that the names instantiating the variables $\boldsymbol{\alpha}$ and $\boldsymbol{\beta}$ must be distinct. A freshness assumption like $(\alpha, x)$, where $x$ is a process variable, records that in any environment the name instantiating $\boldsymbol{\alpha}$ must be fresh for the term instantiating $\boldsymbol{x}$.

Using this auxiliary information, the type system assumes that it is safe to abstract a variable, using lambda abstraction or sum over names, only if no freshness assumptions have been made on it.

The type system of new-HOPLA terms can be specified using judgements of the form:

$$
A ; \Gamma ; d \vdash t: \mathbb{P}
$$

where

- $A \equiv \alpha_{1}: \mathbb{N}, \ldots, \alpha_{k}: \mathbb{N}$ is a collection of name variables;

- $\Gamma \equiv x_{1}: \mathbb{P}_{1}, \ldots, x_{k}: \mathbb{P}_{k}$ is a partial function from process variables to types;

- $d$ is a set of pairs $(\alpha, x) \in A \times \Gamma$, and $(\alpha, \beta) \in A \times A$, keeping track of the freshness assumptions.

Notation: We write $\boldsymbol{d} \backslash \boldsymbol{\alpha}$ for the set of freshness assumptions obtained from $\boldsymbol{d}$ by deleting all pairs containing $\alpha$. The order in which variables appear in a distinction is irrelevant; we will write $(\alpha, \beta) \in d$ as a shorthand for $(\alpha, \beta) \in d \operatorname{or}(\beta, \alpha) \in d$. When we write $\Gamma \cup \Gamma^{\prime}$ we allow the environments to overlap; the variables need not be disjoint provided the environments are consistent.

Actions are typed along the same lines, even if type judgements explicitly report the resumption variable:

$$
A ; \Gamma ; d ; x: \mathbb{R} \Vdash p: \mathbb{P} .
$$

The meaning of the environment $A ; \Gamma ; d$ is exactly the same as above. The variable $x$ is the resumption variable of the pattern $\boldsymbol{p}$, and its type is $\mathbb{R}$.

The type system of new-HOPLA is reported in Table 3 and Table 4.

The rule responsible for generating freshness assumptions is the rule for new-name application. If the term $t$ has been typed in the environment $A ; \Gamma_{;} d$ and $\alpha$ is a newname variable (that is, $\alpha \notin A$ ), then the term $t[\alpha]$ is well-typed under the hypothesis that any name instantiating the variable $\boldsymbol{\alpha}$ is distinct from all the names in terms instantiating the variables that can appear in $\boldsymbol{t}$. This is achieved adding the set of freshness assumptions $\{\alpha\} \times(\Gamma \cup A)$ to $d$ (when convenient, as here, we will confuse an environment with its domain). The rule for pattern matching also modifies the freshness assumptions. The operational rule of pattern matching substitutes a subterm of $t$, whose names are contained in $A^{\prime}$, for $\boldsymbol{x}$. Accordingly, the typing rule initially checks that no name in $\boldsymbol{A}^{\prime}$ belongs to the set of the variables supposed fresh for $\boldsymbol{x}$. Our attention is then drawn to the term $u\left[t^{\prime} / x\right]$, where $t^{\prime}$ is a subterm of $t$. A name variable $\alpha \in A$ 


$$
\begin{aligned}
& \overline{A ; \Gamma ; d ; ; x: \mathbb{R} \Vdash ! x: ! \mathbb{R}} \quad \frac{A ; \Gamma ; d ; ; x: \mathbb{R} \Vdash p: \mathbb{P}}{A ; \Gamma ; d ; ; x: \mathbb{R} \Vdash \alpha \cdot p: \mathbb{N} \otimes \mathbb{P}} \alpha \in A \\
& \frac{\alpha: \mathbb{N}, A ; \Gamma ; d ; ; x: \mathbb{R} \Vdash p: \mathbb{P}}{A ; \Gamma ;(d \backslash \alpha) ; ; x^{\prime}: \delta \mathbb{R} \Vdash n e w \alpha \cdot p\left[x^{\prime}[\alpha] / x\right]: \delta \mathbb{P}} \quad \frac{A ; \Gamma ; d ; ; x: \mathbb{R} \Vdash p: \mathbb{P}}{A ; \Gamma ; d ; ; x: \mathbb{R} \Vdash \alpha \mapsto p: \mathbb{P}} \alpha \in A \\
& \frac{A ; \Gamma ; d \vdash u: \mathbb{Q} \quad A ; \Gamma ; d ; ; x: \mathbb{R} \Vdash p: \mathbb{P}}{A ; \Gamma ; d ; ; x: \mathbb{R} \Vdash u \mapsto p: \mathbb{Q} \rightarrow \mathbb{P}} \quad \frac{A ; \Gamma ; d ; ; x: \mathbb{R} \Vdash p: \mathbb{P}_{j} \quad j \in I}{A ; \Gamma ; d ; ; x: \mathbb{R} \Vdash(j: p): \Sigma_{i \in I} \mathbb{P}_{i}} \\
& \frac{A ; \Gamma ; d ; ; x: \mathbb{R} \Vdash t: \mathbb{P}_{j}[\mu \vec{P} . \overrightarrow{\mathbb{P}} / \vec{P}]}{A ; \Gamma ; d ; ; x: \mathbb{R} \Vdash t: \mu_{j} P: \overrightarrow{\mathbb{P}}} \quad \frac{A ; \Gamma ; d ; ; x: \mathbb{R} \Vdash p: \mathbb{P}}{A^{\prime} ; \Gamma^{\prime} ; d^{\prime} ; ; x: \mathbb{R} \Vdash p: \mathbb{P}} \begin{array}{l}
A \subseteq A^{\prime} \\
d \subseteq d^{\prime}
\end{array}
\end{aligned}
$$

Table 3. new-HOPLA: typing rules for actions

$$
\begin{aligned}
& \overline{A ; \Gamma ; d \vdash \emptyset: \mathbb{P}} \quad \overline{A ; x: \mathbb{P}, \Gamma ; d \vdash x: \mathbb{P}} \quad \frac{A ; \Gamma ; d \vdash t: \mathbb{P}}{A^{\prime} ; \Gamma^{\prime} ; d^{\prime} \vdash t: \mathbb{P}} \underset{d \subseteq d^{\prime}}{\Gamma \subseteq \Gamma^{\prime}} \quad \frac{A ; \Gamma ; d \vdash t: \mathbb{P}}{A ; \Gamma ; d \vdash ! t: ! \mathbb{P}} \\
& \frac{\alpha: \mathbb{N}, A ; \Gamma ; d \vdash t: \mathbb{P}}{A ; \Gamma ; d \vdash \Sigma_{\alpha \in \mathbb{N}} t: \mathbb{P}} \alpha \notin d \quad \frac{\alpha: \mathbb{N}, A ; \Gamma ; d \vdash t: \mathbb{P}}{A ; \Gamma ; d \vdash \lambda \alpha . t: \mathbb{N} \rightarrow \mathbb{P}} \alpha \notin d \quad \frac{A ; x: \mathbb{Q}, \Gamma ; d \vdash t: \mathbb{P}}{A ; \Gamma ; d \vdash \lambda x . t: \mathbb{Q} \rightarrow \mathbb{P}} x \notin d \\
& \frac{\alpha: \mathbb{N}, A ; \Gamma ; d \vdash t: \mathbb{P}}{A ; \Gamma ;(d \backslash \alpha) \vdash \text { new } \alpha . t: \delta \mathbb{P}} \quad \frac{A ; \Gamma ; d \vdash t: \delta \mathbb{P}}{\alpha: \mathbb{N}, A ; \Gamma ; d \cup(\{\alpha\} \times(\Gamma \cup A)) \vdash t[\alpha]: \mathbb{P}} \\
& \frac{A ; \Gamma ; d \vdash t: \mathbb{N} \rightarrow \mathbb{P}}{A ; \Gamma ; d \vdash t \alpha: \mathbb{P}} \alpha \in A \quad \frac{A ; \Gamma ; d \vdash t: \mathbb{P} \rightarrow \mathbb{Q} \quad A ; \Gamma ; d \vdash u: \mathbb{P}}{A ; \Gamma ; d \vdash t u: \mathbb{Q}} \quad \frac{A ; \Gamma ; d \vdash t: \mathbb{P}_{i}}{A ; \Gamma ; d \vdash i: t: \Sigma_{i \in I} \mathbb{P}_{i}} \\
& \frac{A ; \Gamma ; d \vdash t: \Sigma_{i \in I} \mathbb{P}_{i}}{A ; \Gamma ; d \vdash \pi_{i} t: \mathbb{P}_{i}} \quad \frac{A ; x: \mathbb{P}, \Gamma ; d \vdash t: \mathbb{P}}{A ; \Gamma ; d \vdash \operatorname{rec} x . t: \mathbb{P}} x \notin d \quad \frac{A ; \Gamma ; d \vdash t_{i}: \mathbb{P} \quad \forall i \in I}{A ; \Gamma ; d \vdash \Sigma_{i \in I} t_{i}: \mathbb{P}} \\
& \frac{A ; \Gamma ; d \vdash t: \mathbb{P}}{A ; \Gamma ; d \vdash \alpha \cdot t: \mathbb{N} \otimes \mathbb{P}} \alpha \in A \quad \frac{A ; \Gamma ; d \vdash t: \mathbb{N} \otimes \mathbb{P}}{A ; \Gamma ; d \vdash \pi_{\alpha} t: \mathbb{P}} \alpha \in A \quad \frac{A ; \Gamma ; d \vdash t: \mathbb{P}_{j}[\mu \vec{P} . \overrightarrow{\mathbb{P}} / \vec{P}]}{A ; \Gamma ; d \vdash t: \mu_{j} P: \overrightarrow{\mathbb{P}}} \\
& \frac{A^{\prime} ; \Gamma^{\prime} ; d^{\prime} \vdash t: \mathbb{P} \quad A^{\prime} ; \Gamma^{\prime} ; d^{\prime} ; ; x: \mathbb{R} \Vdash p: \mathbb{P} \quad A ; x: \mathbb{R}, \Gamma ; d \vdash u: \mathbb{Q}}{A \cup A^{\prime} ; \Gamma \cup \Gamma^{\prime} ; \bar{d} \vdash[t>p(x) \Rightarrow u]: \mathbb{Q}} A^{\prime} \cap\{\alpha \mid(\alpha, x) \in d\}=\emptyset \\
& \text { where } \bar{d}=(d \backslash x) \cup d^{\prime} \cup\left\{\{\alpha\} \times\left(A^{\prime} \cup \Gamma^{\prime}\right) \mid(\alpha, x) \in d\right\}
\end{aligned}
$$

Table 4. new-HOPLA: typing rules for processes

supposed fresh from $x$ when typing $u$, must now be supposed fresh from all the free variables of $t^{\prime}$. This justifies the freshness assumptions $\left\{\{\alpha\} \times\left(A^{\prime} \cup \Gamma^{\prime}\right) \mid(\alpha, x) \in d\right\}$.

The rest of the type system follows along the lines of type systems for the simply typed $\lambda$-calculus.

The type system assumes that terms do not contain name constants. This is to avoid the complications in a type system coping with both name variables and constants at the same time. We write $s \vdash t: \mathbb{P}$ when there is a judgement $A ; \emptyset ; d \vdash \sigma t^{\prime}: \mathbb{P}$ and a substitution $\sigma$ for $A$ respecting the freshness assumptions $d$ such that $t$ is $\sigma t^{\prime}$. Similarly for patterns. 
Proposition 1 The judgement $\boldsymbol{s} \vdash \boldsymbol{t}: \mathbb{P}$ holds iff there is a canonical judgement $A ; \emptyset ;\{(\alpha, \beta) \mid \boldsymbol{\alpha} \neq \boldsymbol{\beta}\} \vdash \boldsymbol{t}^{\prime}: \mathbb{P}$, in which the substitution $\boldsymbol{\sigma}$ is a bijection between name variables and names and $\boldsymbol{t}$ is $\boldsymbol{\sigma} \boldsymbol{t}^{\prime}$.

We can now prove that the operational rules are type correct.

Lemma 2 (Substitution Lemma) If $A^{\prime} ; \Gamma^{\prime} ; d^{\prime} \vdash t: \mathbb{Q}$ and $A ; x: \mathbb{Q}, \Gamma ; d \vdash u: \mathbb{P}$, where $\Gamma \cup \Gamma^{\prime}$ is consistent and $A^{\prime} \cap\{\alpha \mid(\alpha, x) \in d\}=0$, then $A \cup A^{\prime} ; \Gamma \cup \Gamma^{\prime} ; \bar{d} \vdash$ $u[t / x]: \mathbb{P}$ where $\bar{d}=(d \backslash x) \cup d^{\prime} \cup\left\{\{\alpha\} \times\left(A^{\prime} \cup \Gamma^{\prime}\right) \mid(\alpha, x) \in d\right\}$.

Theorem 3 (Transitions preserve types) If $\vdash t: \mathbb{P}$ and $s ; x: \mathbb{R} \Vdash p: \mathbb{P}$ and $\mathbb{P} ; s \vdash t \stackrel{p(x)}{\longrightarrow} t^{\prime}$, then $s \vdash t^{\prime}: \mathbb{R}$.

\section{Equivalences}

After introducing some notations regarding relations, we explore the bisimulation equivalence that arises from the transition semantics. A relation $\mathcal{R}$ between typing judgements is said to respect types if, whenever $\mathcal{R}$ relates $\boldsymbol{E}_{1} \vdash \boldsymbol{t}_{\mathbf{1}}: \mathbb{P}_{1}$ and $\boldsymbol{E}_{\mathbf{2}} \vdash \boldsymbol{t}_{\mathbf{2}}$ : $\mathbb{P}_{2}$, we have $E_{1} \equiv E_{2}$ and $\mathbb{P}_{1} \equiv \mathbb{P}_{2}$. We are mostly interested in relations between closed terms, and we write $s \vdash t \mathcal{R} u: \mathbb{P}$ to denote $(s \vdash t: \mathbb{P}, s \vdash q: \mathbb{P}) \in \mathcal{R}$.

Definition 4 (Bisimilarity) A symmetric type-respecting relation on closed terms, $\mathcal{R}$, is a bisimulation if whenever $\boldsymbol{s} \vdash t \mathcal{R} u: \mathbb{P}$ and $\mathbb{P} ; \boldsymbol{s}^{\prime} \vdash \boldsymbol{t} \stackrel{\boldsymbol{p}(x)}{\longrightarrow} t^{\prime}$ for $\boldsymbol{s}^{\prime} \supseteq \boldsymbol{s}$, there exists a term $\boldsymbol{u}^{\prime}$ such that $\mathbb{P} ; \boldsymbol{s}^{\prime} \vdash \boldsymbol{u} \stackrel{\boldsymbol{p}(\boldsymbol{x})}{\longrightarrow} \boldsymbol{u}^{\prime}$ and $\boldsymbol{s}^{\prime} \vdash \boldsymbol{t}^{\prime} \mathcal{R} \boldsymbol{u}^{\prime}: \mathbb{R} ;$ where $\mathbb{R}$ is the type of the resumption variable $\boldsymbol{x}$ in $\boldsymbol{p}$. Let bisimilarity, denoted $\sim$, be the largest bisimulation.

We say that two closed terms $\boldsymbol{t}$ and $\boldsymbol{q}$ are bisimilar if $\boldsymbol{s} \vdash \boldsymbol{t} \sim \boldsymbol{q}: \mathbb{P}$ for some $\boldsymbol{s}$ and $\mathbb{P}$.

In the definition of bisimulation, the universal quantification on sets of names $\boldsymbol{s}^{\prime}$ is required, otherwise we would relate $\{a\} \vdash \lambda \alpha \cdot[\alpha ! 0>a ! x \Rightarrow ! 0]: \mathbb{N} \rightarrow ! 0$ and $\{a\} \vdash \lambda \alpha . ! 0: \mathbb{N} \rightarrow ! 0$ while these two terms behave differently in a world where $\boldsymbol{a}$ is not the only current name.

Using an extension of Howe's method [6] as adapted by Gordon and Pitts to a typed setting $[5,10]$, we show that bisimilarity is preserved by well typed contexts.

Theorem 5 Bisimilarity $\sim$ is an equivalence relation and a congruence.

Proposition 2 For closed, well-formed, terms the equations reported in Table 5 hold.

Proposition 3 Bisimilarity validates $\boldsymbol{\beta}$-reduction on new-name abstraction:

$$
s \dot{\cup}\{a\} \vdash(\text { new } \alpha . t)[a] \sim t[a / \alpha]: \mathbb{P} .
$$

\section{Examples}

In this section, we illustrate how new-HOPLA can be used to give semantics to well-known process algebras.

We introduce an useful product type $\mathbb{P} \& \mathbf{Q}$, which is not primitive in new-HOPLA. It is definable as $1: \mathbb{P}+2: \mathbb{Q}$. The projections are given by $f s t(t)=\pi_{1}(t)$ and $\boldsymbol{s n d}(t)=$ $\pi_{2}(t)$, while pairing is defined as $(t, u)=1: t+2: u$. For actions $(p,-)=1: p,(-, q)=$ $2: g$. It is then easy to verify that $s \vdash f s t(t, u) \sim t: \mathbb{P}$, that $s \vdash s n d(t, u) \sim u: \mathbb{Q}$, and that $s \vdash(f s t(t, u)$, snd $(t, u)) \sim(t, u): \mathbb{P} \& \mathbb{Q}$, for all $s \supseteq n(t) \cup n(u)$. 


$$
\begin{array}{rlrl}
s \vdash(\lambda x . t) u & \sim t[u / x]: \mathbb{P} & s \vdash(\lambda \alpha . t) a & \sim t[a / \alpha]: \mathbb{P} \\
s \vdash \lambda x .(t x) & \sim t: \mathbb{P} \rightarrow \mathbb{Q} & s \vdash \lambda \alpha .(t \alpha) & \sim t: \mathbb{N} \rightarrow \mathbb{P} \\
s \vdash \lambda x .\left(\Sigma_{i \in I} t_{i}\right) & \sim \Sigma_{i \in I}\left(\lambda x . t_{i}\right): \mathbb{P} \rightarrow \mathbb{Q} & s \vdash \lambda \alpha .\left(\Sigma_{i \in I} t_{i}\right) & \sim \Sigma_{i \in I}\left(\lambda \alpha . t_{i}\right): \mathbb{N} \rightarrow \mathbb{P} \\
s \vdash\left(\Sigma_{i \in I} t_{i}\right) u & \sim \Sigma_{i \in I}\left(t_{i} u\right): \mathbb{P} & s \vdash\left(\Sigma_{i \in I} t_{i}\right) a & \sim \Sigma_{i \in I}\left(t_{i} a\right): \mathbb{P} \\
s \vdash \pi_{\beta}(\beta \cdot t) & \sim t: \mathbb{P} & s \vdash \pi_{\beta}(\alpha \cdot t) & \sim 0: \mathbb{P} \\
s \vdash \beta \cdot\left(\Sigma_{i \in I} t_{i}\right) & \sim \Sigma_{i \in I} \beta \cdot t_{i}: \mathbb{P} & s \vdash \pi_{\beta}\left(\Sigma_{i \in I} t_{i}\right) & \sim \Sigma_{i \in I} \pi_{\beta} t_{i}: \mathbb{P} \\
s \vdash t & \sim \Sigma_{\alpha \in \mathbb{N} \alpha} \cdot\left(\pi_{\alpha} t\right): \mathbb{N} \otimes \mathbb{P} & s \vdash[u>! x \Rightarrow t] & \sim t[u / x]: \mathbb{P} \\
s \vdash\left[\Sigma_{i \in I} u_{i}>! x \Rightarrow t\right] \sim \Sigma_{i \in I}\left[u_{i}>! x \Rightarrow t\right]: \mathbb{P}
\end{array}
$$

\section{Table 5. Proposition 2: equations}

$\boldsymbol{\pi}$-calculus $\quad$ We denote name constants with $\boldsymbol{a}, \boldsymbol{b}, \ldots$, and name variables with $\boldsymbol{\alpha}, \boldsymbol{\beta}, \ldots$; the letters $n, m, \ldots$ range over both name constants and name variables. The terms of the language are constructed according the following grammar:

$$
P, Q::=0|P| Q|(\nu \alpha) P| \bar{n} m . P \mid n(\alpha) . P \text {. }
$$

The late labelled transition system (denoted $\stackrel{\boldsymbol{\alpha}}{\longrightarrow}$ ) ) and the definition of strong late bisimulation (denoted $\sim_{l}$ ) are standard [13]. ${ }^{2}$

We can specify a type $\mathbb{P}$ as

$$
\mathbb{P}=\tau: ! \mathbb{P}+\text { out } \mathbb{N} \otimes \mathbb{N} \otimes \mathbb{P}+\text { bout: } \mathbb{N} \otimes !(\delta \mathbb{P})+\text { inp: } \mathbb{N} \otimes !(\mathbb{N} \rightarrow \mathbb{P})
$$

The terms of $\boldsymbol{\pi}$-calculus can be expressed in new-HOPLA as the following terms of type $\mathbb{P}$ :

$$
\begin{aligned}
& \llbracket 0 \rrbracket=0 \quad \llbracket \bar{n} m . P \rrbracket=\text { out: } n \cdot m \cdot ! \rrbracket P \rrbracket \quad \llbracket n(\beta) . P \rrbracket=\text { inp: } n \cdot !(\lambda \beta \cdot \llbracket P \rrbracket) \\
& \llbracket(\nu \alpha) P \rrbracket=\operatorname{Res}(\text { newa. } \llbracket P \rrbracket) \quad \llbracket P \mid Q \rrbracket=\llbracket P \rrbracket \| \llbracket Q \rrbracket
\end{aligned}
$$

Here, Res $: \delta \mathbb{P} \rightarrow \mathbb{P}$ and $\|: \mathbb{P} \& \mathbb{P} \rightarrow \mathbb{P}$ (we use infix notation for convenience) and are abbrevations for the recursively defined processes reported in Table 6 .

Informally, the restriction map Res $: \delta \mathbb{P} \rightarrow \mathbb{P}$ pushes restrictions inside processes as far as possible. The five summands correspond to the five equations below:

$$
\begin{gathered}
(\nu \alpha) \tau . P \sim l \tau .(\nu \alpha) P \\
(\nu \alpha) \bar{m} n . P \sim \bar{m} n .(\nu \alpha) P \text { if } \alpha \neq m, n \quad(\nu \alpha) \bar{m} \alpha . P \sim \bar{m}(\alpha) . P \text { if } \alpha \neq m \\
(\nu \alpha) \bar{m}(\beta) . P \sim \bar{m}(\beta) .(\nu \alpha) P \text { if } \alpha \neq m \quad(\nu \alpha) m \beta . P \sim_{l} m \beta .(\nu \alpha) P \text { if } \alpha \neq m
\end{gathered}
$$

where $\bar{m}(\alpha)$ is an abbreviation to express bound-output, that is, $(\boldsymbol{\nu} \alpha) \bar{m} \alpha$. The map Res implicitly also ensures that $(\boldsymbol{\nu} \alpha) P \sim_{l} 0$ if none of the above cases applies. The parallel composition map \| captures the (late) expansion law of $\pi$-calculus. There is a strong correspondence between actions performed by a closed $\pi$-calculus process and the actions of its encoding.

Theorem 6 Let $P$ a closed $\pi$-calculus process. If $P \stackrel{r}{\longrightarrow} l P^{\prime}$ is derivable in $\pi$-calculus, then $n(\llbracket P \rrbracket) \vdash \llbracket P \rrbracket \stackrel{r: 1}{\longrightarrow} t$ for some $t$, and $n(t) \vdash t \sim \llbracket P^{\prime} \mathbb{:}: \mathbb{P}$. Conversely, if

\footnotetext{
${ }^{2}$ To avoid complicating proofs, we ignore replication; that can be encoded as $[! P]=\operatorname{nec} x \cdot([P] \| x)$.
} 


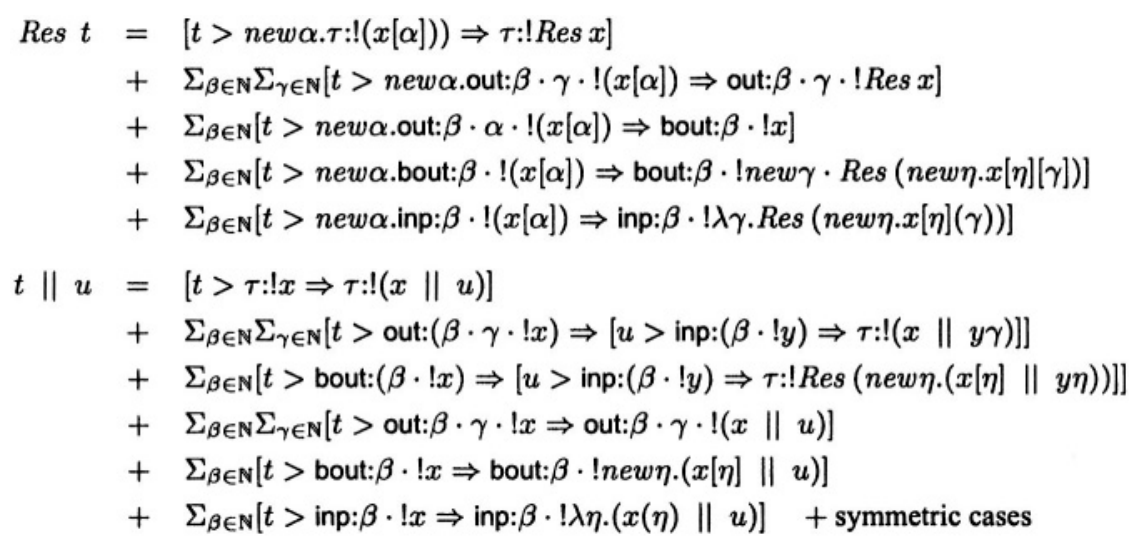

where $\eta$ is chosen to avoid clashes with the free name variables of $u$.

Table 6. Restriction and parallel composition for $\pi$-calculus

$\mathrm{n}(\llbracket P \rrbracket) \vdash \llbracket P \rrbracket \stackrel{\tau: !}{\longrightarrow} t$ in new-HOPLA, then $P \stackrel{\tau}{\rightarrow} P^{\prime}$ for some $P^{\prime}$, and $\mathrm{n}(t) \vdash t \sim$ $\| P^{\prime} \rrbracket: \mathbb{P}$.

The encoding also preserves and reflects late strong bisimulation.

Theorem 7 Let $P$ and $Q$ be two closed $\pi$-calculus processes. If $P \sim_{l} Q$ then $n(P) \cup$ $\mathrm{n}(Q) \vdash \llbracket P \rrbracket \sim \llbracket Q \rrbracket: \mathbb{P}$. Conversely, if $\mathrm{n}(\llbracket P \rrbracket) \cup \mathrm{n}(\llbracket Q \rrbracket) \vdash \llbracket P \rrbracket \sim \llbracket Q \rrbracket: \mathbb{P}$, then $P \sim_{l} Q$.

Along the same lines, new-HOPLA can encode the early semantics of $\pi$-calculus. The type of the input action assigned to $\pi$-calculus terms captures the difference between the two semantics. In the late semantics a process performing an input action has type inp: $\mathbb{N} \otimes !(\mathbb{N} \rightarrow \mathbb{P})$ : the type of the continuation $(\mathbb{N} \rightarrow \mathbb{P})$ ensures that the continuation is actually an abstraction that will be instantiated with the received name when interaction takes place. In the early semantics, the type of a process performing an input action is changed into Inp: $\mathbb{N} \otimes \mathbb{N} \rightarrow ! \mathbb{P}$. Performing an input action now involves picking up a name before executing the prototypical action, and in the continuation (whose type is $\mathbb{P}$ ) the formal variable has been instantiated with the received name. Details can be found in [16].

Higher-Order $\boldsymbol{\pi}$-calculus The language we consider can be found in [13]. Rather than introducing a unit value, we allow processes in addition to abstractions to be communicated. For brevity, we gloss over typing issues. The syntax of terms and values is defined below.

$$
P::=V \bullet V|n(x) . P| \bar{n}(V) . P|P| P|x|(\nu \alpha) P|0 \quad V::=P|(x) . P
$$

The reduction semantics for the language is standard [13]; we only recall the axioms that define the reduction relation:

$$
(x) . P \bullet V \rightarrow P[V / x|\quad \bar{n}(V) . P| n(x) \cdot Q \rightarrow P \mid Q[V / x] .
$$

Types for $\mathbf{H O} \pi$ are given recursively by

$$
\mathbb{P}=\tau: ! \mathbb{P} \text { +oul: } \mathbb{N} \otimes ! \mathbb{C}+\text { inp: } \mathbb{N} \otimes !(\mathbb{F} \rightarrow \mathbb{P}) \quad \mathbb{C}=0: \mathbb{F} \& \mathbb{P}+1: \delta \mathbb{C} \quad \mathbf{F}=2: \mathbb{P}+3: \mathbb{F} \rightarrow \mathbb{P} .
$$


Concretions of the form $(\boldsymbol{\nu} \tilde{\alpha})\langle V\rangle P$ correspond to terms of type $\mathbb{C}$; recursion on types is used to encode the tuple of restricted names $\tilde{\alpha}$. Thefunctions $\llbracket-\rrbracket_{v}$ and $\llbracket-\rrbracket$ translate respectively values into the terms of type $\mathbb{F}$, and processes into terms of type $\mathbb{P}$ :

$$
\begin{aligned}
& \llbracket P \rrbracket_{v}=2: \llbracket P \rrbracket \quad \llbracket(x) \cdot P \rrbracket_{v}=3: \lambda x \cdot \llbracket P \rrbracket \quad \llbracket V \bullet W \rrbracket=\tau: !\left(\pi_{3} \llbracket V \rrbracket_{v}\right)\left(\pi_{2} \llbracket W \rrbracket_{v}+\pi_{3} \llbracket W \rrbracket_{v}\right) \\
& \llbracket P \mid Q \rrbracket=\llbracket P \rrbracket \| \llbracket Q \rrbracket \quad \llbracket(\nu \alpha) P \rrbracket=\text { Res new } \mathbb{\| P \rrbracket} \quad \llbracket x \rrbracket=x \quad \llbracket 0 \rrbracket=0 \\
& \llbracket n(x) \cdot P \rrbracket=\text { inp: } n \cdot !(\lambda x \cdot \llbracket P \rrbracket) \quad \llbracket \bar{n}(V) \rrbracket=\text { out: } n \cdot !(\llbracket V \rrbracket v, \llbracket P \rrbracket) .
\end{aligned}
$$

The restriction map Res $: \delta \mathbb{P} \rightarrow \mathbb{P}$ filters the actions that a process emits, blocking actions that refer to the name that is being restricted. Output actions cause names to be extruded: the third summand records these names in the appropriate concretion.

$$
\begin{aligned}
& \text { Res } t=[t>\text { new } \alpha . \tau: ! x[\alpha] \Rightarrow \tau: \mid \text { Res } x] \\
& +\Sigma_{\beta \in \mathbb{N}}[t>\text { new } \alpha \cdot \text { inp: }(\beta \cdot ! x \mid \alpha]) \Rightarrow \text { inp: }(\beta \cdot \mid \lambda y \cdot \text { Res }(\text { new } \gamma \cdot x[\gamma \mid(y)))] \\
& +\Sigma_{\beta \in \mathbb{N}}[t>\text { new } \alpha . \text { out: }(\beta \cdot\lfloor x \mid \alpha]) \Rightarrow \text { out: }(\beta \cdot ! 3: x)]
\end{aligned}
$$

Parallel composition is a family of mutually dependent operations also including components such as $\|_{\boldsymbol{i}}$ of type $\mathbb{C} \& \mathbb{F} \rightarrow \mathbb{P}$ to say how values compose in parallel with concretions etc. All these components can be tupled together in a product and parallel composition defined as a simultaneous recursive definition:

- Processes in parallel with processes:

$$
\begin{aligned}
t \| u & =\Sigma_{\beta \in \mathrm{N}}\left[t>\text { out: } \beta \cdot ! x \Rightarrow\left[u>\text { inp: } \beta \cdot ! y \Rightarrow \tau: !\left(x \|_{i} y\right)\right]\right] \\
& +\Sigma_{\beta \in \mathrm{N}}\left[u>\text { inp: } \beta \cdot ! y \Rightarrow \text { inp: } \beta \cdot !\left(t \|_{a} y\right)\right] \\
& +\Sigma_{\beta \in \mathrm{N}}\left[u>\text { out: } \beta \cdot ! y \Rightarrow \text { out: } \beta \cdot !\left(t \|_{c} y\right)\right] \\
& +\{u>\tau: ! y \Rightarrow \tau: !(t \| y)] \quad+\text { symmetric cases }
\end{aligned}
$$

- Concretions in parallel with values

$$
\begin{aligned}
c \|_{i} f= & \operatorname{snd}\left(\pi_{0} c\right) \|\left(\left(\pi_{3} f\right)\left(\pi_{2}\left(f_{s t}\left(\pi_{0} c\right)\right)+\pi_{3}\left(f s t\left(\pi_{0} c\right)\right)\right)\right. \\
& \left.+\operatorname{Res}\left(\text { newa. }\left(\left(\left(\pi_{1} c\right)[\alpha]\right) \|_{i} f\right)\right)\right)
\end{aligned}
$$

- Concretions in parallel with processes

$$
c \|_{c} t=0:\left(f s t\left(\pi_{0} c\right), \operatorname{snd}\left(\pi_{0} c\right) \| t\right)+1:\left(\text { new } \alpha .\left(\left(\pi_{1} c\right)[\alpha] \|_{c} t\right)\right)
$$

- Values in parallel with processes

$$
f \|_{a} t=\lambda x .\left(\left(\left(\pi_{3} f\right) x\right) \| u\right)
$$

The remaining cases are given symmetrically. The proposed encoding agrees with the reduction semantics of $\mathrm{HO} \pi$. The resulting bisimulation is analogous to the so called higher-order bisimulation [1, 15], and as such it is strictly finer than observational equivalence. It is an open problem whether it is possible to provide an encoding of $\mathrm{HO} \pi$ that preserves and reflects the natural observational equivalence given in [12].

Polyadic $\pi$-calculus A natural and convenient extension to $\pi$-calculus is to admit processes that pass tuples of names: polyadicity is a good testing ground for the expressivity of our language. We can specify a type for polyadic $\pi$-calculus processes as:

$\mathbb{P}=\tau: ! \mathbb{P}+$ out $: \mathbb{N} \otimes \mathbb{C}+$ inp $: \mathbb{N} \otimes \mathbb{E} \quad \mathbb{C}=0: \mathbb{N} \otimes \mathbb{C}+1: \delta \mathbb{C}+2: ! \mathbb{P} \quad \mathbb{F}=3: \mathbb{N} \rightarrow \mathbb{F}+4: \mathbb{P}$ 
Recursive types are used to encode tuples of (possibly new) names in concretions, and sequences of name abstractions in abstractions.

Just as with the $\pi$-calculus, it is possible to write a restriction map Res $: \delta \mathbb{P} \rightarrow \mathbb{P}$ that pushes restrictions inside processes as far as possible, and a parallel map that captures the expansion law. The resulting semantics coincides with the standard late semantics of polyadic $\pi$-calculus. Details can be found in [16].

Mobile Ambients We sketch an encoding of the mobility core of the Ambient Calculus, extending the encoding of Mobile Ambients with public names into HOPLA given in [7], Details can be found in [16].

Types reflect the actions that ambient processes can perform, and are given recursively by:

$$
\begin{aligned}
& \mathbb{P}=\tau: ! \mathbb{P}+\text { in: } \mathbb{N} \otimes ! \mathbb{P}+\text { out: } \mathbb{N} \otimes ! \mathbb{P}+\text { open: } \mathbb{N} \otimes ! \mathbb{P}+\text { mvin: } \mathbb{N} \otimes \mathbb{C}
\end{aligned}
$$

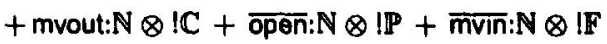

$$
\begin{aligned}
& \mathbb{C}=0: \mathbb{P} \& \mathbb{P}+1: \delta \mathbb{C} \quad \mathbb{F}=\mathbb{P} \rightarrow \mathbb{P}
\end{aligned}
$$

The injections in, out, and open correspond to the basic capabilities a process can exercise, while their action on the enclosing ambients is registered by the components mvin and mvout. The injections open and $\overline{\mathbf{m v i n}}$ record the receptive interactions that an ambient can (implicitly) have with the environment. Again, recursive types are used in concretions to record the sequence of names that must be extruded. Terms are then translated as:

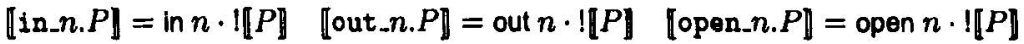

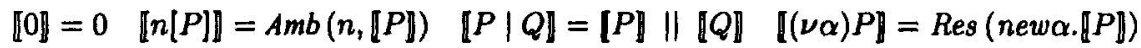

The restriction map Res $: \delta \mathbb{P} \rightarrow \mathbb{P}$ filters the actions that a process emit, and blocks actions that refer to the name that is restricted. In fact, in Mobile Ambients, the only scope extrusions are caused by mobility, and not by pre-actions.

$$
\text { Res } t=\cdots+\Sigma_{\beta \in \mathbb{N}}[t>\text { new } \alpha \cdot \ln :(\beta \cdot \mid x[\alpha]) \Rightarrow \text { in: }(\beta \cdot ! \operatorname{Res} x)]+\cdots
$$

Parallel composition is a family of operations, one of which is a binary operation be-

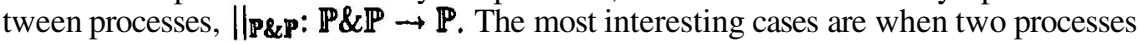
interact:

$$
\begin{aligned}
& t \| u=\Sigma_{\beta \in N}\left[t>\text { open: } \beta \cdot ! x \Rightarrow\left[u>\text { open: } \beta \cdot ! y \Rightarrow \tau: !\left(x \|_{c} y\right)\right]\right] \\
& \left.+\Sigma_{\beta \in N}|t>\overline{m v i n}: \beta \cdot| f \Rightarrow\left[u>\text { mvin: } \beta \cdot l c \Rightarrow \tau: ! l\left(c \|_{i} f\right)\right]\right]+\cdots
\end{aligned}
$$

Interaction between concretions, abstractions, and processes is analogous to that in the $\mathrm{HO} \pi$ encoding. Finally, ambient creation can be defined recursively in new-HOPLA as an operation $A m b: \mathbb{N} \& \mathbb{P} \rightarrow \mathbb{P}$;

$$
\begin{aligned}
& \operatorname{Amb}(m, t)=[t>\tau: ! x \Rightarrow \tau: \operatorname{lAmb}(m, x)] \\
& +\Sigma_{\beta \in \mathrm{N}}[t>\operatorname{in}: \beta \cdot \mid x \Rightarrow \operatorname{mvin}: \beta \cdot !(A m b(m, x), 0) \\
& +\Sigma_{\beta \in N}[t>\text { out: } \beta \cdot ! x \Rightarrow \text { mvout: } \beta \cdot !(A m b(m, x), 0) \\
& +[t>\text { mvout: } m \cdot l c \Rightarrow \tau: ! \operatorname{Extr}(m, c)\} \\
& +\overline{\text { open }}: m \cdot 1 t+\overline{\mathrm{mvmn}}: m \cdot \mid \lambda y \cdot A m b(m, t \| y)
\end{aligned}
$$

where the map Extr: $\mathbb{N} \& \mathbf{C} \rightarrow \mathbb{P}$ extrudes names across ambient's boundary after a mvout action:

$$
\operatorname{Extr}(m, c)=f s t\left(\pi_{0} c\right) \| A m b\left(m, \operatorname{snd}\left(\pi_{0} c\right)\right)+\operatorname{Res}\left(\text { newa. }\left(\operatorname{Extr}\left(m,\left(\pi_{1} c\right)[\alpha]\right)\right)\right) .
$$




\section{Conclusion}

This paper has concentrated on the operational semantics of new-HOPLA, which despite its economy has been shown to be remarkably expressive. This is in part because only two of the usual process-algebra operations appear as primitives in newHOPLA: a basic prefix operation and nondeterministic sum. The denotational semantics of new-HOPLA and the domain theories on which they rest will be explained more fully elsewhere. The path-set semantics sketched in the introduction suggests an analysis of adequacy and full abstraction, based on the basic observation of !-transitions, along the lines of $[8,9]$. The more detailed presheaf semantics supports bisimulation, though at higher-order we do not understand how open-map bisimulation, intrinsic to presheaf models, relates to the bisimulation we have defined-in the case of the $\pi$ calculus the two bisimulations agree by [2]. Closer to the concerns of this paper are questions of exploiting the rich types of new-HOPLA to give 'fully-abstract' encodings of higher-order process calculi.

\section{References}

[1] G. Boudol. Towards a lambda calculus for concurrent and communicating systems. In Proc. TAPSOFT '89, volume 351 of LNCS, pages 149-161. Springer Verlag, 1989.

[2] G. L. Cattani, I. Stark, and G. Winskel. Presheaf models for the $\pi$-calculus. In Proc. CTCS'97, volume 1290 of LNCS. Springer Verlag, 1997.

[3] M. Fiore, E. Moggi, and D. Sangiorgi. A fully-abstract model for the $\pi$-calculus. In Proc. 11th LICS. IEEE Computer Society Press, 1996.

[4] J.Y.Girard. Linear logic. Theoretical Computer Science, 50:1-102,1987.

[5] A. D. Gordon. Bisimilarity as a theory of functional programming: mini-course. Notes Series BRICS-NS-95-3, BRICS, Department of CS, University of Aarhus, July 1995.

[6] D. J. Howe. Proving congruence of bisimulation in functional programming languages. Information and Computation, 124(2):103-112,1996.

[7] M. Nygaard and G. Winskel. Hopla-a higher-order process language. In Proc. CONCUR '02, volume 2421 of $L N C S$. Springer Verlag, 2002.

[8] M. Nygaard and G. Winskel. Domain theory for concurrency. To appear in Theoretical Computer Science, special issue on domain theory, accepted 2003.

[9] M. Nygaard and G. Winskel. Full abstraction for HOPLA. In Proc. CONCUR '03, LNCS. Springer Verlag, 2003.

[10] A. M. Pitts. Operationally-based theories of program equivalence. In P. Dybjer and A. M. Pitts, editors, Semantics and Logics of Computation, Publications of the Newton Institute, pages 241-298. Cambridge University Press, 1997.

[11] A, M. Pitts and M. J. Gabbay. A metalanguage for programming with bound names modulo renaming. In Proc. MPC 2000, volume 1837 of LNCS. Springer Verlag, 2000.

[12] D. Sangiorgi. Bisimulation in higher-order calculi. In Proc. IFIP PROCOMET'94, pages 207-224. North-Holland, 1994.

[13] D. Sangiorgi and D. Walker. The $\pi$-calculus: a Theory of Mobile Processes. Cambridge University Press, 2001.

[14] I. Stark. A fully-abstract domain model for the $\pi$-calculus. In Proc. 11th LICS. IEEE Computer Society Press, 1996.

[15] B. Thomsen. Calculi for Higher Order Communicating Systems. PhD thesis, Department of Computing, Imperial College, 1990.

[16] F. Zappa Nardelli. De la sémantique des processus d'ordre supérieur. $\mathrm{PhD}$ thesis, Université de Paris 7, 2003. Available in English from http: / /www. di . ens. fr/ zappa. 